\title{
Atherosclerosis Influences the Vasomotor Response of Epicardial Coronary Arteries to Exercise
}

\author{
John B. Gordon, Peter Ganz, Elizabeth G. Nabel, R. David Fish, Jose Zebede, Gilbert H. Mudge, \\ R. Wayne Alexander, and Andrew P. Selwyn \\ Cardiovascular Division, Department of Medicine, Brigham and Women's Hospital, Harvard Medical School, \\ Boston, Massachusetts 02115
}

\begin{abstract}
We studied the vasomotion of epicardial coronary arteries during exercise and tested the hypotheses that abnormal vasoconstriction is related to the presence of atherosclerosis and may be related to endothelial dilator dysfunction. During cardiac catheterization quantitative coronary angiography was performed in 21 patients during supine bicycle exercise. 21 of 28 smooth, angiographically normal vessel segments dilated (14.0 $\pm 1.8 \%)$ during exercise; four smooth segments did not change whereas only three constricted. In contrast, 15 of 16 vessel segments with irregularities constricted in response to exercise (17.0 $\pm 0.1 \%)$ with only one segment dilating. All 10 stenotic segments constricted to exercise $(23 \pm 4 \%)$. Six patients also received intracoronary acetylcholine before exercise to test endothelium-dependent dilator function. In five of six patients all nine vessel segments showed the same directional response to acetylcholine and exercise. Three irregular and two stenotic segments constricted with acetylcholine $(51 \pm 21 \%)$ and exercise $(9.0 \pm 0.6 \%)$. In contrast, four smooth segments dilated to acetylcholine $(19 \pm 6 \%)$ and exercise $(9 \pm 1 \%)$; Both exercise and acetylcholine generally dilated smooth but constricted irregular and stenosed coronary segments. It appears likely that atherosclerosis plays an important role in the abnormal vasomotion of diseased coronary arteries during exercise and the pattern of abnormality suggests impairment of vasodilator function.
\end{abstract}

\section{Introduction}

There is growing interest in the disturbed vasomotion of diseased coronary arteries particularly in relation to activities that are well known to cause transient myocardial ischemia. Recent studies indicate that atherosclerosis may disturb vasomotor function and stenosed coronary arteries may actively reduce coronary blood flow (1-3). However, the mechanism(s) responsible for coronary vasomotor changes during exercise have not been clearly defined.

In this study we examined the response of coronary artery segments to supine bicycle exercise in 15 consecutive patients and demonstrated the constriction of atherosclerotic segments

Address reprint request to Dr. Gordon, Cardiovascular Division, Department of Medicine, Brigham and Women's Hospital, 75 Francis Street, Boston, MA 02115.

Received for publication 28 April 1988 and in revised form 6 January 1989.

J. Clin. Invest.

(c) The American Society for Clinical Investigation, Inc.

0021-9738/89/06/1946/07 \$2.00

Volume 83, June 1989, 1946-1952 with minor luminal irregularity or stenoses. Only angiographically normal, smooth segments dilated to exercise. We have previously shown that acetylcholine, an endothelium-dependent vasodilator, also causes paradoxical constriction of atherosclerotic epicardial coronary arteries and dilates smooth vessels indicating that atherosclerosis leads to loss of endothelium dependent vasodilator function (4). This vasomotor response in relation to the angiographic appearance of the coronary arteries was seen in patients undergoing exercise in this study and suggested the hypothesis that the vasoconstriction of diseased coronary arteries to exercise may be related to atherosclerosis and to loss of the endothelium-dependent vasodilator mechanism. To test this hypothesis, we examined the response of the same coronary segments to exercise and intracoronary acetylcholine in six additional consecutive patients.

\section{Methods}

\section{Patient selection}

21 patients referred for diagnostic cardiac catheterization to assess the extent of coronary artery disease were recruited. These patients were divided into three groups based on angiographic evidence of atherosclerosis documented on the diagnostic angiograms. Classification was made before analysis by consensus of two or three investigators who were blinded to the study results:

Group I: smooth vessels. Six patients had entirely smooth, angiographically normal-appearing coronary arteries. There were four men and two women. All patients had atypical chest pain and a negative exercise tolerance test. The mean age was $52 \pm 7$ yr with a range of 39-58 yr.

Group II: minimal disease. Four patients had luminal irregularity of one or more coronary arteries with focal narrowing that did not exceed $30 \%$ of the vessel diameter. There were three men and one woman with a mean age of $50 \pm 8 \mathrm{yr}$ and a range of 38-54 yr. Each patient had troublesome chest pain that was not clearly precipitated by exercise and had a negative or nondiagnostic exercise tolerance test.

Group III: advanced disease. 11 patients, 10 men and 1 woman, had a stenosis in at least one coronary artery $\geq 50 \%$. Each patient had stable exertional angina and a positive standard Bruce exercise tolerance test highly predictive of significant coronary artery disease defined as 2-mm or greater ST segment depression, or 1-mm ST segment depression and angina (5). The mean age for this group of patients was $54 \pm 7 \mathrm{yr}$ with a range of 43-64 yr.

Patients with unstable angina, Prinzmetal's variant angina, diabetes mellitus, myocardial infarction within $3 \mathrm{mo}$, or congestive heart failure were excluded. Written informed consent was obtained separately for the research protocol and for the diagnostic cardiac catheterization in accordance with the guidelines established by the Committee for the Protection of Human Subjects at the Brigham and Women's Hospital.

\section{Study protocol}

Beta-blocking drugs were discontinued four half-lives before study. Calcium blockers and long-acting nitrates were held for at least $12 \mathrm{~h}$ 
before angiography. Sublingual nitroglycerin was unrestricted until $1 \mathrm{~h}$ before catheterization.

Diagnostic right and left heart catheterization was performed using either a right brachial artery cut down $(n=8)$ or the standard percutaneous femoral approach $(n=13)$. 15-45 min after the diagnostic catheterization, all patients studied began an exercise protocol. A 7 French Judkins type catheter or 7F Amplatz catheter was introduced into the left main $(n=13)$ or right coronary artery $(n=2)$. A 7 French Swan-Ganz catheter was placed in the pulmonary capillary wedge position. The heart rate (beats per minute), blood pressure (millimeters of mercury), electrocardiogram (ECG), ' and mean pulmonary capillary wedge pressure (PCW) (millimeters of mercury) were continuously measured.

By means of test injections, the coronary arteries under study were positioned at the measured isocenter of a Polydiagnost $\mathrm{C}$ biplane system (Philips Medical Systems, Inc., Shelton, CT) and 7-9 ml of nonionic contrast (iohexol, Omnipaque, Winthrop-Breon, Inc., New York) were injected at $6 \mathrm{ml} / \mathrm{s}$ at a maximum of $150 \mathrm{psi}$ using a Medrad Power Injector $(3,4,6)$. Repeat coronary angiograms were obtained while heart rate, blood pressure, the ECG, and the PCW were continuously measured. Supine exercise was performed using an ergometer bicycle (Uniwork, model 845, Quinton Instrument Co., Seattle, WA). Baseline measurements were recorded before exercise (control). Exercise was initiated at a work level of $200-400 \mathrm{~kg}-\mathrm{m} / \mathrm{min}$ and resistance was increased by $200 \mathrm{~kg}-\mathrm{m} / \mathrm{min}$ every $2 \mathrm{~min}$. Peak exercise was defined as the point at which the patient developed significant fatigue, ST segment depression, or angina. All measurements were repeated at peak of exercise, $1 \mathrm{~min}$ after exercise, and after $40 \mu \mathrm{g}$ of intracoronary nitroglycerin infused over $4 \mathrm{~min}$.

Venous blood samples for plasma norepinephrine and epinephrine were drawn at rest, peak exercise, and 1 min postexercise. Plasma norepinephrine and epinephrine concentrations were determined by the radioenzymatic assay described by Peuler and Johnson (7).

Because of the pattern of reactions of smooth and diseased coronary segments in the first 15 patients, the next six consecutive patients received intracoronary acetylcholine before the exercise protocol described above. The protocol for delivering acetylcholine has been described previously $(3,4)$. Briefly, after the completion of the diagnostic catheterization, an additional 5,000 $\mathrm{U}$ of heparin was administered and a No. 8 French L-4 Judkins type guiding catheter was introduced into the left main coronary artery. A No. 2 French coronary infusion catheter (American Edwards, Santa Ana, CA, or Advanced Catheter System, Mountain View, CA) was advanced through the guiding catheter into the proximal portion of the left anterior descending artery for administration of acetylcholine. A No. 5 French Bipolar pacing catheter was placed in the right ventricular apex and set in a demand mode to prevent the heart rate from slowing to below 40 beats $/ \mathrm{min}$.

Serial 2-min intracoronary infusions of acetylcholine were administered in the following sequence: control 1 (5\% dextrose and water); vehicle control 2 ( $5 \%$ dextrose and water plus $0.005 \%$ manitol); graded concentrations of acetylcholine (to achieve estimated final blood concentrations in the left anterior descending coronary of $10^{-9}, 10^{-8}, 10^{-7}$, and $\left.10^{-6} \mathrm{M}\right)$; and repeat control (5\% dextrose and water). The final coronary blood concentration of acetylcholine was estimated with the assumption that the blood flow in the vessel was $80 \mathrm{ml} / \mathrm{min}$ (8). Acetylcholine infusions were terminated either when the largest dose $\left(10^{-6}\right.$ M) was reached or when obvious vessel narrowing or occlusion occurred. Infusions were carried out with a pump (Harvard Apparatus Co., Inc., S. Natick, MA) to keep the flow rates under $1 \%$ of the estimated coronary blood flow. At the end of each infusion coronary arteriography was performed with the use of a power injection of nonionic contrast as described above. The heart rate, systemic arterial pressure, and ECG (leads 1, AVF, V2) were monitored continuously.

No complications were encountered during these infusions or the exercise tests.

1. Abbreviations used in this paper: ECG, electrocardiogram; PCW, mean pulmonary capillary wedge pressure.

\section{Analysis of coronary arteriograms}

In order to examine the serial reactions of the available coronary segments, the technically more suitable single-plane coronary angiograms were selected from the biplane views for analysis. Segments of the proximal, midvessel, and distal portions of either the left anterior descending artery, circumflex, or right coronary artery were inspected and two or three segments were chosen for quantitative analysis in each patient if they showed the following characteristics: $(a) \geq 5 \mathrm{~mm}$ in length, $(b)$ easily identifiable using side branches as anatomical landmarks, and $(c)$ free of overlapping vessels. Once a coronary segment was selected and analyzed none were rejected from this study. The technique for the quantitative analysis of coronary artery diameters has been described in detail $(3,4,6,9)$. For each coronary injection, four cine frames were selected from end-diastole in one cycle when the coronary artery showed full homogeneous opacification. The epicardial coronary artery segment under study was centered, cine frames were digitized and averaged by the use of a video camera (Cohu, Inc., San Diego, CA) and a computer (Microvax II, Digital Equipment Corp., Marlboro, MA) connected to a video interface (Recognition Concepts, Inc., Incline Village, NV) and image processor. Multiple videoscans and two-line profile averaging were used to minimize noise. Calibrated grids in the field of view were used to scale the data from pixels to millimeters. A series of measurements of diameter were recorded for each pixel line for the length of the arterial segment, and the data were also displayed in the form of a graph showing average diameter versus length for the four cine frame estimates at each diameter measured. Fixed coordinates were used to reproduce anatomical regions of interest in repeated measurements to assess serial changes.

\section{Statistical analysis}

For each subject, the digitized cine frames for each treatment were summed and averaged along the segment profile to give a mean diameter and standard deviation at each point. A single mean diameter for the segment for each treatment was obtained by averaging this measure along the segment profile. These segment responses were grouped by treatment for subjects with smooth, irregular, and stenotic vessels. Where an analysis of variance showed significant variation among treatment responses pairwise $t$ tests (in two tails) were applied to compare treatment groups according to the Bonferroni procedure so that the summed errors of measurement did not exceed 0.05 , allowing rejection of the null hypothesis at the 0.95 confidence level for each comparison. Previous validation of our techniques $(3,4,5,9)$ has indicated that the minimum detectable change of diameter is $5 \%$. Therefore, this threshold was used to identify segment responses as dilation, constriction, or no change.

The differences in directional responses to peak exercise and response to acetylcholine infusion were tested using the Kappa statistic (10). Changes in systemic hemodynamics were compared using the paired $t$ test. All values are expressed as mean \pm SEM.

\section{Results}

\section{Systemic hemodynamics and catecholamine response}

The rate pressure product, $\mathrm{PCW}$, and catecholamine levels increased during peak supine bicycle exercise in all 21 patients. These data are summarized for each group in Table I. These hemodynamic results also show that the rate pressure product and the PCW return to control within $1 \mathrm{~min}$ after exercise. However, in all of the groups the plasma levels of norepinephrine and epinephrine remained elevated at one minute after exercise at a level that was not significantly different from the peak measurements (Table I).

None of the group I or group II patients experienced angina and/or ST segment changes during the exercise protocol whereas eight of the 11 patients with an advanced stenosis (group III) did so. 
Table I. Systemic Hemodynamics and Catecholamine Response

\begin{tabular}{|c|c|c|c|c|}
\hline & Control & Peak exercise & One-minute post-exercise & NTG \\
\hline \multicolumn{5}{|l|}{ Group I: smooth vessels only } \\
\hline Rate pressure product $\left(\mathrm{HR} \times \mathrm{SBP} \times 10^{3}\right)$ & $9.1 \pm 0.42$ & $16.3 \pm 0.59^{8}$ & $10.5 \pm 0.63$ & $7.9 \pm 0.74$ \\
\hline $\mathrm{PCW}(\mathrm{mmHg})$ & $10.6 \pm 0.93$ & $19.0 \pm 2.6^{\ddagger}$ & $9.8 \pm 1.4$ & $6.8 \pm 1.5$ \\
\hline Norepinephrine $(\mathrm{pg} / \mathrm{ml})$ & $177.3 \pm 19$ & $425.2 \pm 68^{*}$ & $344.3 \pm 55.6^{*}$ & \\
\hline Epinephrine $(\mathrm{pg} / \mathrm{ml})$ & $40.1 \pm 8.2$ & $125.4 \pm 44$ & $70.0 \pm 25.4$ & \\
\hline \multicolumn{5}{|l|}{ Group II: minimal disease } \\
\hline Rate pressure product & $8.7 \pm 1.3$ & $14.6 \pm 1.9^{\ddagger}$ & $11.5 \pm 1.5$ & $9.02 \pm 0.79$ \\
\hline PCW $(m m H g)$ & $8.3 \pm 1.5$ & $14.3 \pm 5$ & $5.5 \pm 1.2$ & $5.0 \pm 0.58$ \\
\hline Norepinephrine $(p g / m l)$ & $122.6 \pm 21$ & $242 \pm 10^{*}$ & $198.0 \pm 8.5$ & \\
\hline Epinephrine $(p g / m l)$ & $39.2 \pm 4.9$ & $91 \pm 31$ & $57.7 \pm 25.8$ & \\
\hline \multicolumn{5}{|l|}{ Group III: advanced stenoses } \\
\hline Rate pressure product & $10.4 \pm 0.66$ & $15.8 \pm 0.77^{\S}$ & $11.9 \pm 0.80$ & $10.1 \pm 0.35$ \\
\hline $\mathrm{PCW}(\mathrm{mmHg})$ & $11.8 \pm 1.2$ & $28.4 \pm 3.2^{\S}$ & $12.8 \pm 2.7$ & $6.8 \pm 1.2$ \\
\hline Norepinephrine $(\mathrm{pg} / \mathrm{ml})$ & $185.2 \pm 54$ & $347.6 \pm 42^{\ddagger}$ & $348.3 \pm 37^{\S}$ & \\
\hline Epinephrine $(p g / m l)$ & $80.0 \pm 24$ & $113.1 \pm 32^{\ddagger}$ & $100.2 \pm 22$ & \\
\hline
\end{tabular}

Results are expressed as mean $\pm \mathrm{SE}$. Significance of data when compared with control measurements: ${ }^{*} P 0.05,{ }^{\ddagger} P 0.01,{ }^{\S} P 0.001$. HR, heart rate in beats per minute; SBP, systolic blood pressure; PCW, mean pulmonary capillary wedge pressure; NTG, nitroglycerin.

There was no significant change in heart rate or mean blood pressure in five of the six patients receiving acetylcholine. The mean heart rate and systolic blood pressures were $72 \pm 5$ beats/min and $128 \pm 7 \mathrm{mmHg}$ at rest and $70 \pm 5$ beat $/ \mathrm{min}$ and $128 \pm 1 \mathrm{mmHg}$ with the highest dose of acetylcholine. One patient experienced transient occlusion of the left anterior descending artery in response to $10^{-6} \mathrm{M}$ acetylcholine. This was associated with ST segment elevation, followed by a slowing of the heart rate from 96 beats/min at rest to 66 beats/min after acetylcholine, and a drop in blood pressure from 150/95 $\mathrm{mmHg}$ at rest to $123 / 78 \mathrm{mmHg}$. The vessel occlusion, ST segment elevation, and the associated hemodynamic changes resolved promptly (within seconds) with discontinuation of the acetylcholine infusion.

\section{Angiographic appearance and the reaction of coronary segments to exercise}

Fig. 1 is an illustrative example of three coronary segments from one patient showing dilation with exercise in a smooth segment and constriction of irregular and stenosed segments.

Group I: smooth vessels only. 15 angiographically normal segments were analyzed in the six patients in group $I$ and overall showed dilation during exercise by $11 \pm 3 \%(P<0.01)$, (Fig. 2). On inspection of the individual responses, $11 \mathrm{seg}$ ments dilated during exercise from a mean control diameter of $1.8 \pm 0.1$ to $2.1 \pm 0.1 \mathrm{~mm}$, a $16 \pm 3 \%$ increase. Three segments in one patient showed no change during exercise while another segment from this patient constricted from 1.7 to $1.6 \mathrm{~mm}$, an $8 \%$ decrease in vessel diameter. All 15 of these smooth segments dilated in response to $40 \mu \mathrm{g}$ of intracoronary nitroglycerin from a mean control diameter of $1.8 \pm 0.1$ to $2.6 \pm 0.2 \mathrm{~mm}$, a $35 \pm 5 \%$ increase $(P<0.01)$.

Group II: minimal disease. 12 coronary segments were analyzed in four patients with intimal irregularity of one or more coronary arteries but no luminal narrowing $>30 \%$ (Fig. 3) Seven of the segments analyzed had intimal irregularity while five segments were smooth. All seven irregular segments constricted during exercise from a mean vessel diameter of
$2.0 \pm 0.4$ to $1.8 \pm 0.3 \mathrm{~mm}(P<0.005)$, a decrease of $14 \pm 3.5 \%$. Of the five smooth vessel segments, three dilated to exercise from a mean vessel diameter of 2.2 to $2.4 \mathrm{~mm}$. One smooth vessel did not change with exercise although another smooth segment constricted from $4.0 \mathrm{~mm}$ during control to $3.4 \mathrm{~mm}$ at peak exercise, a $17 \%$ decrease. All segments dilated to intracoronary nitroglycerin. The irregular segments dilated from a mean control diameter of $2.1 \pm 0.4$ to $2.4 \pm 0.4 \mathrm{~mm}(P<0.05)$ after nitroglycerin, a $19 \pm 4 \%$ increase, while the smooth segments dilated from $2.6 \pm 0.4$ to $3.2 \mathrm{~mm}(P<0.01)$, a $27 \pm 12 \%$ increase in vessel diameter.

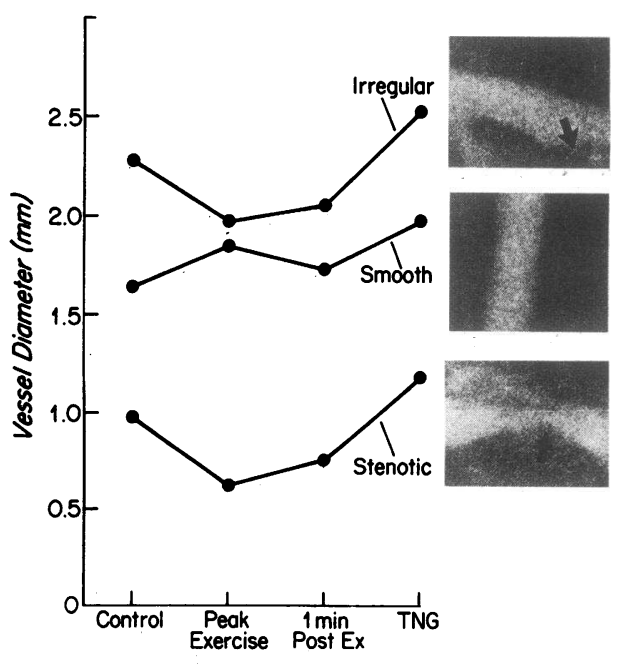

Figure 1. This illustration is taken from one patient who had an important stenosis of a large obtuse marginal branch of the left circumflex coronary artery, an irregular proximal left anterior descending artery, and a smooth distal obtuse marginal. Exercise caused dilation of the smooth segment and constriction of the irregular and stenosed coronary segments. This example illustrates the heterogeneous vasomotor response of coronary artery segments to exercise that appear to be related to the angiographic extent of coronary atherosclerosis. 


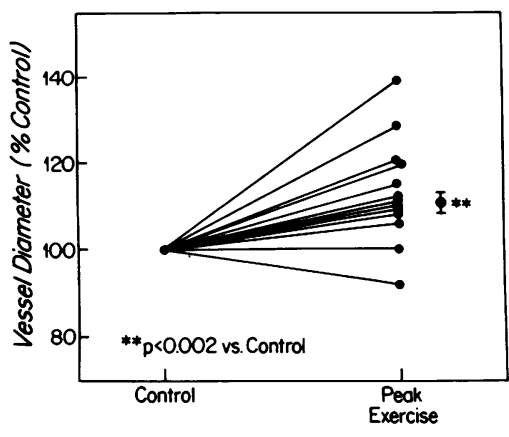

Figure 2. The vasomotor response of coronary artery segments from a group of patients with entirely smooth coronary arteries at arteriography. The majority of segments dilated during exercise, whereas three remained unchanged and one constricted. The overall response for all segments was significant dilation.

Group III: advanced stenoses. 28 coronary segments were analyzed in the 11 patients with at least one single stenosis $>50 \%$ : 11 segments were stenotic, 9 were irregular, and 8 were smooth. One stenotic segment could not be accurately measured due to the extreme severity of narrowing and was excluded from analysis. All 10 of the stenotic segments analyzed constricted with exercise (Fig. 4). The mean diameter for stenotic segments decreased from $1.1 \pm 0.2 \mathrm{~mm}$ during control to $0.9 \pm 0.2 \mathrm{~mm}(P<0.001)$ at peak exercise, a constriction of $23 \pm 4 \%$. Similarly, eight of nine irregular segments constricted to exercise while only one segment dilated. The eight irregular segments that constricted narrowed from a control diameter of $1.8 \pm 0.1$ to $1.6 \pm 0.1 \mathrm{~mm}(P \leqslant 0.005)$ at peak exercise, an average constriction of $8 \pm 2 \%$. Conversely, seven of eight smooth segments dilated to exercise while one constricted. The eight smooth segments dilated $10 \pm 0.3 \%$ increasing vessel diameter from $2.1 \pm 0.2 \mathrm{~mm}$ at control to $2.3 \pm 0.2 \mathrm{~mm}(P<0.02)$ at peak exercise (Fig. 4).

All 28 vessel segments dilated in response to $40 \mu \mathrm{g}$ of intracoronary nitroglycerin. The mean diameter of the stenotic segments increased $18 \pm 9 \%$ from $1.1 \pm 1.6 \mathrm{~mm}$ control to $1.2 \pm 0.1 \mathrm{~mm}(P<0.02)$, irregular segments dilated $18 \pm 5 \%$ from $1.9 \pm 0.6 \mathrm{~mm}$ in control to $2.2 \pm 0.2 \mathrm{~mm}(P<0.002)$, and the smooth segments dilated $32 \pm 6 \%$ from a control diameter of $2.1 \pm 0.2$ to $2.7 \pm 0.2 \mathrm{~mm}(P<0.001)$ after intracoronary nitroglycerin.

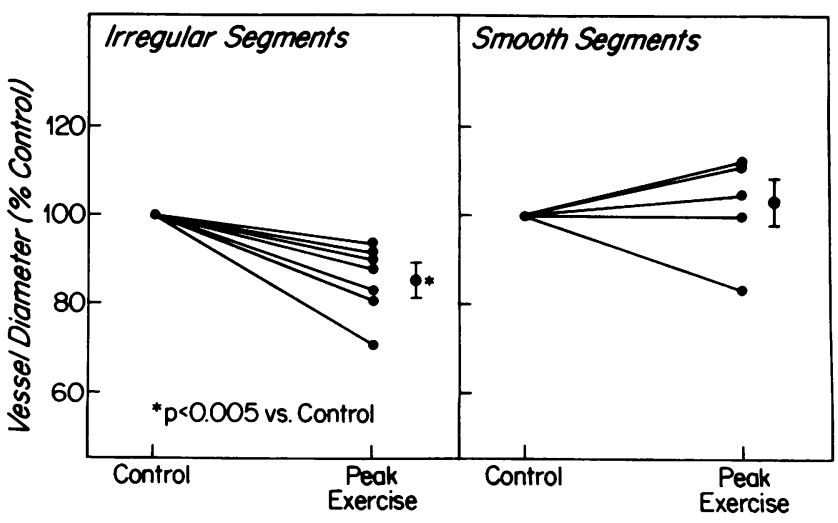

Figure 3. The vasomotor response of the coronary artery segments from a group of patients with luminal irregularities but no stenoses seen at coronary arteriography. All irregular segments constricted with exercise. In contrast, three of the five smooth segments dilated, one smooth segment remained unchanged, and one smooth segment constricted.

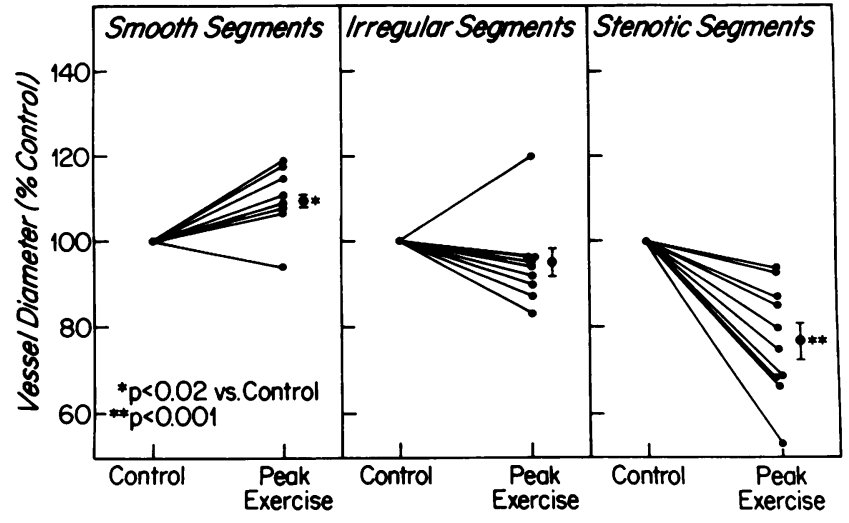

Figure 4. In this third group of patients, the vasomotor responses of smooth, irregular, and stenosed coronary segments were assessed during exercise. All of the stenosed segments and all but one of the irregular coronary artery segments constricted with exercise. In contrast, all but one of the smooth coronary segments dilated with exercise. This group demonstrates the full range of heterogeneous responses of coronary artery segments to exercise demonstrating normal dilation of smooth vessels but constriction in early and late coronary atherosclerosis.

The vasomotor responses to exercise of all the coronary segments from the three groups were combined according to angiographic appearance (Fig. 5). Of 28 smooth segments, 21 dilated from $2.0 \pm 0.1 \mathrm{~mm}$ in control to $2.2 \pm 0.1 \mathrm{~mm}$ at peak exercise, an increase in diameter of $14 \pm 2 \%$. The diameter of four smooth segments did not change with exercise while three smooth segments constricted $10 \pm 2 \%$ from a control mean diameter of 2.5 to $2.1 \mathrm{~mm}$ at peak exercise. Conversely, $16 \mathrm{~min}$ imally diseased, irregular segments constricted from a control diameter of $1.9 \pm 0.2$ to $1.6 \pm 0.2 \mathrm{~mm}(P<0.001)$ at peak exercise, a $17.0 \pm 0.1 \%$ decrease. Only 1 of 16 irregular segments dilated to exercise. All 10 of the advanced stenoses analyzed constricted to exercise. In the 10 stenotic segments the control diameter of $1.1 \pm 0.2 \mathrm{~mm}$ decreased to $0.9 \pm 0.2 \mathrm{~mm}$ at peak exercise, a change of $23 \pm 4 \%$. All vessels dilated in response to intracoronary nitroglycerin (smooth segments, $33 \pm 4 \%$; irregular segments, $18 \pm 3.4 \%$; and stenotic segments, $18 \pm 9 \%$ ).

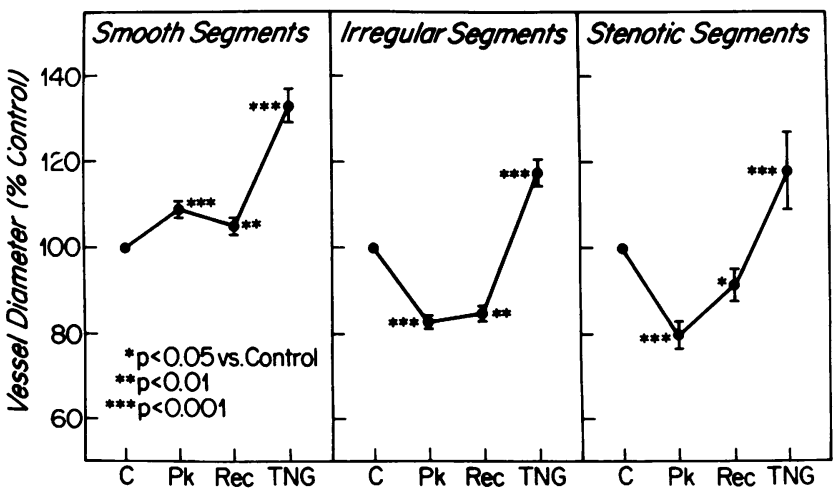

Figure 5. The separate vasomotor responses to exercise of all smooth, all irregular, and all stenosed coronary segments. Again, the results show dilation of smooth coronary segments but constriction of irregular and stenosed segments with exercise. These data also indicate that, at $1 \mathrm{~min}$ after exercise, the vasomotor responses have not yet returned to control. All coronary segments dilated in response to the intracoronary administration of nitroglycerin. 


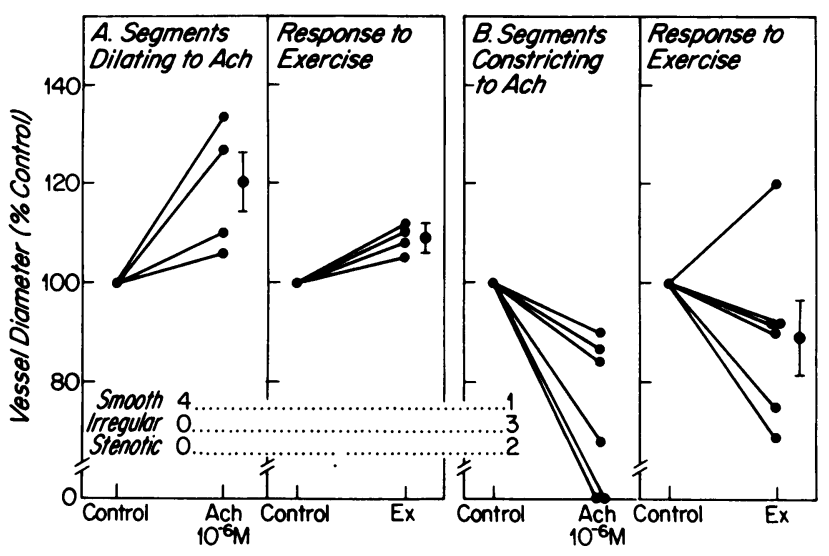

Figure 6. The vasomotor responses of smooth, irregular, and stenosed coronary segments to both intracoronary administration of acetylcholine and exercise. When coronary artery segments showed endothelium-dependent dilation to acetylcholine, they also showed dilation during exercise. In contrast, all of the irregular and stenosed coronary segments demonstrated failure of endothelium-dependent dilation and paradoxical constriction to acetylcholine. These segments also showed abnormal constriction to exercise. One smooth coronary segment constricted in response to acetylcholine but dilated to exercise.

There were no correlations between changes in double product or plasma catecholamines and the direction or extent of vasomotor change of the coronary segments.

\section{Response to acetylcholine and exercise}

The vasomotor responses of six left anterior descending arteries and one large diagonal branch to acetylcholine and exercise were compared in six patients. In five of six patients, all nine segments analyzed showed the same response to these two stimuli. Four smooth segments dilated to both acetylcholine $10^{-6} \mathrm{M}$ and peak exercise ( $19 \pm 6 \%$ and $9 \pm 1 \%$, respectively). The control diameter of $2.1 \pm 0.3 \mathrm{~mm}$ increased to $2.5 \pm 0.3 \mathrm{~mm}$ after acetylcholine and dilated from a recontrol diameter of $2.2 \pm 0.2$ to $2.4 \pm 0.3 \mathrm{~mm}$ at peak exercise. In contrast, three irregular segments and two stenotic segments constricted to acetylcholine $10^{-6} \mathrm{M}$ and exercise $(51 \pm 21 \%$ and $9.0 \pm 0.6 \%$, respectively) (Fig. 6). The control diameter of the irregular segments decreased from $1.9 \pm 0.6$ to $1.2 \pm 1.1 \mathrm{~mm}$ after acetylcholine (one segment occluded) and to $1.7 \pm 0.6 \mathrm{~mm}$ at peak exercise. Similarly, the two stenotic segments constricted from control diameters of 0.5 and $1.3 \mathrm{~mm}$ to occlusion and $1.1 \mathrm{~mm}$ after acetylcholine, respectively, and constricted to $0.4 \mathrm{~mm}$ and $1.0 \mathrm{~mm}$ at peak exercise, respectively (see Fig. 6). All segments dilated to nitroglycerin. The smooth segments dilated $46 \pm 13 \%$; irregular and stenosed segments dilated $46 \pm 14 \%$.

The response was divergent in only one of six patients. In this case the smooth angiographically normal left anterior descending artery constricted in response to acetylcholine $10^{-6}$ $M(16 \%)$ but dilated during exercise (20\%). The Kappa statistic for these six patients receiving acetylcholine before exercise demonstrated significant agreement (0.7) in the direction of the vasomotor responses. Since statistical significance was achieved despite the small number of patients studied and the results were in agreement with previous studies using acetylcholine in patients $(3,4)$, additional patients were not recruited for this lengthy and demanding protocol.

\section{Discussion}

This study has demonstrated that the epicardial coronary arteries in man exhibit a range of reactions to exercise: smooth segments dilated while segments with early (irregularities) and late (stenosis) angiographic evidence of atherosclerosis constricted. Not only was this disturbed vasomotion associated with the presence of atherosclerosis but the pattern of reactions in the first $\mathbf{1 5}$ patients was the same as that seen after the local administration of acetylcholine (4). In five of six consecutive patients that followed, each coronary segment showed the same response to exercise and acetylcholine. Although this correlation does not prove cause and effect, these data suggest that endothelium dependent dilation occurs during exercise and endothelial dysfunction in early and late atherosclerosis permits paradoxical constriction.

Gage et al. (11) have observed that only stenotic coronary segments constrict during exercise and this vasoconstriction can be prevented by pretreatment with nitroglycerin. Our results differ in that constriction was not confined to stenoses but also occurred in nearly all irregular segments during exercise.

\section{Possible mechanisms governing dilation of smooth coronary arteries}

The dilation of smooth coronary arteries during exercise may be due to a combination of factors that includes elevated perfusion pressure, formation of vasodilatory metabolites, an increase in $\beta$-adrenergic stimulation, and the release of vasoactive substance(s) by a healthy vascular endothelium.

Vatner et al. (12) have demonstrated in the intact dog that beta-adrenergic mechanisms can mediate an increase in coronary blood flow and an increase in cross sectional area of epicardial coronary arteries. We (6) have recently shown in man that smooth coronary segments dilate with sympathetic nerve stimulation induced by the cold pressor test while vessels with irregularities and stenoses constrict. The dilation of smooth segments was only partially inhibited by $1 \mathrm{mg}$ of intracoronary propranolol. These data would suggest that $\beta$ adrenergic mechanisms are only partly responsible for the dilation of smooth coronary arteries.

A large number of studies in isolated arteries (13-15), intact animals (16-18), and humans $(3,4)$ have confirmed that a functionally intact endothelium is required for vessel relaxation with acetylcholine. There is some controversy in that a limited number of in vitro and in vivo studies have shown constriction of some apparently normal coronary arteries to acetylcholine (19-22). The lack of consistency in the in vitro studies may be due to vascular trauma and endothelial damage as well as use of concentrations of acetylcholine that are high enough to override endothelium-dependent dilation. In addition, in vitro vessel preparations are not exposed to increased blood flow induced by acetylcholine which also mediates endothelium-dependent vasodilation (12). In vivo studies describing constriction of smooth coronary arteries in response to acetylcholine have used single bolus injections of acetylcholine. Bolus injection does not test a dose response and may result in high (beyond $10^{-6} \mathrm{M}$ ) local concentrations of acetylcholine, which will certainly override endothelium-derived vasodilation and directly constrict vascular smooth muscle. Furchgott and colleagues (13) clearly showed in their experiments that endothelium-dependent dilation in normal arteries 
only occurs within a particular concentration range which must be carefully achieved in vitro and in vivo.

In addition, increases in pulsatile flow $(17,23,24)$, norepinephrine (16), serotonin (16), and many other agonists are known to dilate or limit constriction of normal coronary arteries via an endothelium-dependent mechanism $(16,17,20,24)$. Interestingly, norepinephrine activates specific alpha-2 receptors on the endothelial cell that attenuate norepinephrine-induced contraction (16).

This study has also shown that the majority of smooth vessel segments dilated to exercise, and four of five smooth segments dilated to both exercise and acetylcholine. One smooth segment dilated to exercise but constricted to acetylcholine. Angiography may have underestimated early atherosclerosis in this case. In the setting of minimal disease, the artery may still dilate in response to exercise but manifest failure of relaxation to acetylcholine due to early impairment of cholinergic relaxation related to atherosclerosis (25).

\section{Possible mechanisms causing constriction}

The precise mechanism(s) of vasoconstriction observed in epicardial coronary arteries is controversial (26). The possibility of passive vascular collapse at the stenosis has been raised (11, 26). However, this study showed constriction of the majority of irregular segments in vessels without significant stenoses (seven segments) suggesting for the first time that passive collapse due to diminished distending pressure is not the predominant mechanism for constriction during exercise. In this exercise study the patients with angiographically normal and diseased coronary arteries all showed an increase in circulating catecholamines. However, the smooth vessel segments showed dilation whereas diseased arteries constricted. Clearly, in the normal vessels the constrictor effects of catecholamines are overcome resulting in dilation.

Considerable experimental evidence from intact animal and human studies indicates that atherosclerosis is associated with enhanced vasoconstriction in response to catecholamines, serotonin, histamine, and ergonovine (15, 27, 28, 29). It is possible that the coronary constriction during exercise seen in atherosclerotic arteries occurred because of increased sensitivity of the smooth muscle to catecholamines or other agonists. In addition, an impaired responsiveness of atherosclerotic vascular smooth muscle cells to stimuli which lead to vasodilation might be present. Decreased responsiveness to nitroglycerin was suggested in this study when all vessel segments were analyzed by their angiographic appearance. The 28 smooth segments dilated considerably more $(33 \pm 4 \%)$ than the 16 irregular segments $(18 \pm 3.4 \%)$ and the 10 stenotic segments $(18 \pm 9 \%)$. This apparent difference in the response to nitroglycerin between normal and atherosclerotic vessels could be due to atherosclerotic changes in the vessel wall (fibrosis, lipid deposition, calcification) as well as primary smooth muscle cell dysfunction.

Recent angiographic studies in patients have demonstrated dilation of normal coronary segments to acetylcholine but constriction of minimally diseased or stenotic vessels $(3,4)$. These earlier studies in man have shown that endothelium-dependent dilation exists in normal vessels but is replaced by paradoxical constriction in both early and advanced atherosclerosis. The results of this study showed that exercise resulted in dilation of smooth segments but constriction of irregular and stenosed segments, a pattern similar to that seen after acetylcholine infusion. It is possible that early and advanced coronary atherosclerosis causes disturbance in endotheliumdependent vasomotor function. As a result the normal dominating dilator effect of healthy endothelium is lost in the diseased state and constrictor influences may predominate as seen in this study.

\section{Clinical implications}

Vasoconstriction during exercise in diseased coronary arteries could further limit myocardial oxygen supply particularly when superimposed on a critical coronary artery stenosis. In addition, this paradoxical constriction might be a useful functional indicator of active atherosclerosis even in a minimally diseased vessel. Its reversal, leading to appropriate dilator responses, might parallel regression of atherosclerosis. This concept is supported by primate studies (30) but human data are currently lacking.

In summary, the dilatation or constriction of epicardial coronary arteries with exercise appears to be related to the presence of the angiographic evidence of atherosclerosis and is mirrored by the response of the vessel to the endothelium-dependent vasodilator acetylcholine. The loss of normal dilation and appearance of abnormal constriction seen during exercise in diseased vessels may be related to dysfunction of endothelium-dependent dilation in atherosclerosis. These observations may have important implications for better understanding the pathophysiology of transient myocardial ischemia and how it relates to the biology of atherosclerosis.

\section{Acknowledgments}

Dr. Ganz is a Clinical Investigator of the National Heart, Lung and Blood Institute (NHLBI) (HL-01045). Dr. Fish is a Physician-Scientist of the NHLBI (HL-01957). This research was supported by National Institutes of Health grant (HL-35295) and a grant from the Ciba Geigy Foundation.

\section{References}

1. Brown, B. G., E. L. Bolson, and H. T. Dodge. 1984. Dynamic mechanisms in human coronary stenosis. Circulation. 70:917-922.

2. Deanfield, J. E., M. Shea, P. Ribiero, C. M. deLandsheere, R. A. Wilson, P. Horlock, and A. P. Selwyn. 1984. Transient ST-segment depression as a marker of myocardial ischemia during daily life. Am.J. Cardiol. 54:1195-1200.

3. Fish, R. D., E. G. Nabel, A. P. Selwyn, P. L. Ludmer, G. H. Mudge, J. M. Kirshenbaum, F. J. Schoen, R. W. Alexander, and P. Ganz. 1988. Responses of coronary arteries of cardiac transplant patients to acetylcholine. J. Clin. Invest. 81:21-31.

4. Ludmer, P. L., A. P. Selwyn, T. L. Shook, R. R. Wayne, G. H. Mudge, R. W. Alexander, and P. Ganz. 1986. Paradoxical vasoconstriction induced by acetylcholine in atherosclerotic coronary arteries. N. Engl. J. Med. 315:1046-1051.

5. Blomquist, C. G. 1971. Use of exercise testing for diagnostic and functional evaluation of patients with arteriosclerotic heart disease. Circulation. 44:1120-1136.

6. Nabel, E. G., P. Ganz, J. B. Gordon, R. W. Alexander, and A. P. Selwyn. 1988. Dilation of normal and constriction of atherosclerotic coronary arteries caused by the cold pressor test. Circulation. 77:4352.

7. Peuler, J. D., and G. A. Johnson. 1977. Simultaneous single isotope radioenzymatic of plasma norepinephrine, epinephrine and dopamine. Life Sci. 21:625-636.

8. Ganz, W., K. Tamura, H. S. Marcus, R. Donoso, S. Yoshida, 
and H. J. C. Swan. 1971. Measurement of coronary sinus blood flow by continuous thermodilution in man. Circulation. 44:181-195.

9. Spears, J. R., T. Sandor, A. V. Als, M. Malagold, J. E. Markis, W. Grossman, J. R. Serur, and S. Paulin. 1983. Computerized image analysis for quantitative measurement of vessel diameter from cineangiograms: computerized image for quantitative measurement of vessel diameter from cineangiograms. Circulation. 68:453-461.

10. Cohen, J. 1968. Weighted Kappa: nominal scale agreement with provision for scaled disagreement or partial credit. Am. Psychol. Bull. 70:213-218.

11. Gage, J. E., O. M. Hess, T. Murakami, M. Ritter, J. Grimm, and $H$. P. Krayenbuehl. 1986. Vasoconstriction of stenotic coronary arteries during dynamic exercise in patients with classic angina pectoris: reversibility by nitroglycerin. Circulation. 73:865-876.

12. Vatner, S. F., T. H. Hintze, and P. Macho. 1982. Regulation of large coronary arteries by beta-adrenergic mechanisms in the conscious dog. Circ. Res. 51:56-66.

13. Furchgott, R. F., and J. V. Zawadzki. 1980. The obligatory role of endothelial cells in the relaxation of arterial smooth muscle by acetylcholine. Nature (Lond.). 288:373-376.

14. Furchgott, R. F. 1980 . Role of endothelium in responses of vascular smooth muscle. Circ. Res. 53:557-573.

15. Freiman, P. C., G. G. Mitchell, D. D. Heistad, M. L. Armstrong, and D. D. Harrison. 1986. Atherosclerosis impairs endothelium-dependent vascular relaxation to acetylcholine and thrombin in primates. Circ. Res. 58:783-789.

16. Cocks, T. M., and J. A. Angus. 1983. Endothelium-dependent relaxation of coronary arteries by noradrenaline and serotonin. Nature (Lond.). 305:627-630.

17. Pohl, V., S. Holtz, R. Busse, and E. Bassenge. 1986. Crucial role of endothelium in the vasodilator response to increased flow in vivo. Hypertension. 8:37-44.

18. Busse, R., G. Trogisch, and E. Bassenge. 1986. The role of the endothelium in the control of vascular tone. Basic Res. Cardiol. 80:475-490.

19. Horjo, Y., H. Yasue, M. Rokutanda, M. Nokamura, H. Ogawa, K. Takaoka, K. Matsuyama, and T. Kimura. 1986. Effects of intracoronary injection of acetylcholine on coronary arterial diameter. Am. J. Cardiol. 57:984-989.
20. Ku, D., and J. Caulfield. 1987. Human coronary aortic bypass grafts, but not coronary arteries, responded to acetylcholine with endothelium dependent vasorelaxation. Circulation. 76:IV-382. (Abstr.)

21. Matsuyama, K., H. Yasue, K. Okumore, K. Goto, and Y. Morikami. 1987. Different effects of actylcholine on coronary arterial diameters between the young and old humans. Circulation. 76:IV383. (Abstr.)

22. Forstermann, U., A. Mugge, and J. C. Frolich. 1986. Endothelium-dependent relaxation of human epicardial coronary arteries: frequent lack of effect of acetylcholine. Eur. J. Pharmacol. 128:277-281.

23. Hintze, T. H., and S. F. Vatner. 1984. Reactive dilation of large coronary arteries in conscious dogs. Circ. Res. 54:50-57.

24. Holtz, J., U. Forstermann, U. Pohl, M. Giesler, and E. Bassenge. 1984. Flow-dependent, endothelium-mediated dilation of epicardial coronary arteries in conscious dogs: effects of cyclooxygenase inhibition. J. Cardiovasc. Pharmacol. 6:1161-1169.

25. Bossaller, C., G. B. Habib, H. Yamamoto, C. Williams, S. Wells, and P. O. Henry. 1987. Impaired muscarinic endothelium-dependent relaxation and cyclic guanosine 5 '-monophosphate formation in atherosclerotic human coronary artery and rabbit aorta. J. Clin. Invest. 79:170-174.

26. Klein, L. W., B. L. Segal, and R. H. Helfant. 1987. Dynamic coronary stenosis behavior in classic angina pectoris: active process or passive response? J. Am. Coll. Cardiol. 10:311-313.

27. Heistad, D. D., M. L. Armstrong, M. L. Marcus, D. J. Piegors, and A. L. Mark. 1984. Augmented responses to vasoconstrictor stimuli in hypercholesterolemic and atherosclerotic monkeys. Circ. Res. 54:711-718.

28. Shimokawa, H., H. Tomoike, S. Nabeyama, H. Yamamoto, $H$. Araki, and M. Nakamura. 1983. Coronary artery spasm induced in atherosclerotic miniature swine. Science (Wash. DC). 221:560-562.

29. Schroeder, J. S., J. L. Bolen, R. A. Quint, D. A. Clark, W. G. Hayden, C. B. Higgins, and L. Wexler. 1977. Provocation of coronary spasm with ergonovine maleate. Am. J. Cardiol. 40:487-491.

30. Harrison, D. G., P. C. Freiman, M. L. Armstrong, and D. D. Heistad. 1986. Improvement of receptor-mediated endotheliumdependent vascular relaxation following regression of atherosclerosis. Circulation. 74:(Suppl. ii):286. 